\title{
Curative resection of an aldosteronoma causing primary aldosteronism in the second trimester of pregnancy
}

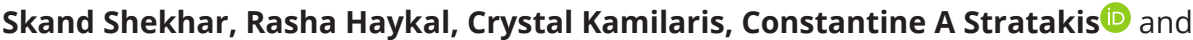 \\ Fady Hannah-Shmouni
}

Section on Endocrinology and Genetics, The Eunice Kennedy Shriver National Institute of Child Health and Human Development, National Institutes of Health, Bethesda, Maryland, USA

\author{
Correspondence \\ should be addressed \\ to F Hannah-Shmouni \\ Email \\ fady.hannah-shmouni@nih. \\ gov
}

\section{Summary}

A 29-year-old primigravida woman with a known history of primary aldosteronism due to a right aldosteronoma presented with uncontrolled hypertension at 5 weeks of estimated gestation of a spontaneous pregnancy. Her hypertension was inadequately controlled with pharmacotherapy which lead to the consideration of surgical management for her primary aldosteronism. She underwent curative right unilateral adrenalectomy at 19 weeks of estimated gestational age. The procedure was uncomplicated, and her blood pressure normalized post-operatively. She did, however, have a preterm delivery by cesarean section due to intrauterine growth retardation with good neonatal outcome. She is normotensive to date.

\section{Learning points:}

- Primary aldosteronism is the most common etiology of secondary hypertension with an estimated prevalence of 5-10\% in the hypertensive population.

- It is important to recognize the subtypes of primary aldosteronism given that certain forms can be treated surgically.

- Hypertension in pregnancy is associated with significantly higher maternal and fetal complications.

- Data regarding the treatment of primary aldosteronism in pregnancy are limited.

- Adrenalectomy can be considered during the second trimester of pregnancy if medical therapy fails to adequately control hypertension from primary aldosteronism.

\section{Background}

Hypertension in pregnancy is a relatively common occurrence that is consistently associated with poor maternal and fetal outcomes (1). Secondary hypertension caused by endocrine dysfunction is less frequent, estimated to comprise less than $2 \%$ of all hypertension in pregnancy (1). Physiological hormonal changes that occur in pregnancy make establishing the diagnosis of endocrine hypertension such as that due to primary aldosteronism (PA) challenging, with less than 100 such cases reported in the literature (2). We report a case of PA in pregnancy due to an aldosterone producing adrenocortical adenoma that was successfully treated with unilateral adrenalectomy in the second trimester after the failure of medical therapy.

\section{Case presentation}

A 29-year-old woman from Ghana with a known history of resistant hypertension and hypokalemia was 
evaluated at the National Institutes of Health (NIH) Clinical Center under protocol 00-CH-0160 (Study of Adrenal Gland Tumors, NICHD, NIH, ClinicalTrials.gov Identifier: NCT00005927) for PA. She was diagnosed with hypertension at the age of 27 and was initiated on treatment with anti-hypertensive medications; however, she had progressive hypertension and hypokalemia to $3 \mathrm{mmol} / \mathrm{L}$ (3.4-5.1 $\mathrm{mmol} / \mathrm{L})$. She moved to the United States 11 months prior to her NIH visit and at the time adjustments were made to her anti-hypertensive therapy but secondary causes of hypertension were not evaluated. About 7 months later, she was found to have significantly elevated blood pressure with systolic blood pressure greater than $200 \mathrm{mmHg}$ and diastolic blood pressure greater than $110 \mathrm{mmHg}$ during evaluation by a reproductive endocrinologist for infertility. She was referred to a local emergency department for further care and was admitted to the hospital. Upon discharge from this hospital, she sought an assessment with a new healthcare provider who detected worsening hypertension and hypokalemia and initiated evaluation for PA. That workup led to her initial diagnosis of PA and referral to the NIH.

Her other medical conditions included elevated Lp(a) and infertility due to blocked fallopian tubes. She did not have a history of nicotine, alcohol or other recreational drug use and her family history was significant for late-onset hypertension in both parents and maternal grandparents. Her physical examination was significant for stage 2 hypertension (based on blood pressure readings), but was otherwise unremarkable, without physical signs of hypercortisolemia, including obesity, facial fullness, dorsocervical or supraclavicular fat deposition, facial plethora, acne, hirsutism, alopecia, thin skin, bruising, striae, muscle weakness, or impaired memory. At the time of her initial evaluation at our Clinical Center, her medications included labetalol, nifedipine, hydrochlorothiazide, losartan, and potassium chloride. To facilitate biochemical evaluation for PA per our research protocol, her anti-hypertensive medications were switched to hydralazine, verapamil, and terazosin.

\section{Investigation}

At her initial pre-pregnancy visit, she underwent biochemical screening for PA with plasma renin activity and plasma aldosterone concentration, which was diagnostic for PA. As per our research protocol, she had additional testing with saline suppression test, which was also consistent with PA (data not shown). She did not have biochemical evidence of hypercortisolism, with the 1-mg overnight dexamethasone suppression test and serial urinary free cortisol measurements within the normal range, and pheochromocytoma was biochemically excluded (Table 1). A CT of the adrenal glands revealed a low attenuation nodule in her right adrenal gland measuring $1.6 \mathrm{~cm} \times 1.0 \mathrm{~cm}$, with a density of $8 \mathrm{HU}$ pre-contrast consistent with a benign adenoma (Fig. 1A and B). An MRI of the adrenal glands showed a hypoenhancing, isointense $\mathrm{T} 1$, hypointense $\mathrm{T} 2$ right adrenal nodule measuring $2.2 \mathrm{~cm} \times 1.0 \mathrm{~cm}$ with signal loss in out of phase sequence, and a normal left adrenal gland (Fig. 1C). As part of the research protocol, she underwent an uncomplicated adrenal venous sampling which localized her disease to the right adrenal gland (data not shown). Following her adrenal venous sampling, she was discharged on the following anti-hypertensive therapy: verapamil $240 \mathrm{mg}$ twice daily, terazosin $5 \mathrm{mg}$ twice daily, hydralazine $50 \mathrm{mg}$ every $8 \mathrm{~h}$ and potassium chloride 40 mEq daily.

\section{Treatment}

Given the patient's unilateral PA, she was referred for unilateral right adrenalectomy and was counselled regarding contraception prior to surgical treatment. The patient returned 8 weeks later for the planning of the definitive surgical management of her PA, but she was

Table 1 Laboratory evaluation of the patient.

\begin{tabular}{|c|c|c|}
\hline $\begin{array}{l}08: 00 \mathrm{~h} \text { (fasting) } \\
\text { laboratory parameter }\end{array}$ & $\begin{array}{l}\text { Normal } \\
\text { range }\end{array}$ & $\begin{array}{l}\text { Measured } \\
\text { value }\end{array}$ \\
\hline Sodium, $\mathrm{mmol} / \mathrm{L}$ & $136-145$ & 141 \\
\hline Potassium, mmol/L & $3.4-5.1$ & 2.6 \\
\hline Chloride, $\mathrm{mmol} / \mathrm{L}$ & $98-107$ & 98 \\
\hline Blood urea nitrogen, $\mathrm{mg} / \mathrm{dL}$ & $6-20$ & 10 \\
\hline Serum creatinine, $\mathrm{mg} / \mathrm{dL}$ & $0.51-0.95$ & 0.60 \\
\hline Plasma glucose, mg/dL & $74-106$ & 110 \\
\hline Bicarbonate, mEq/L & $22-24$ & 30 \\
\hline $\begin{array}{l}\text { Plasma aldosterone } \\
\text { concentration, ng/dL }\end{array}$ & $0-30$ & 26.2 \\
\hline Plasma renin activity, $\mathrm{ng} / \mathrm{mL} / \mathrm{h}$ & $0.6-4.3$ & $<0.15$ \\
\hline Serum cortisol* $\mu \mathrm{g} / \mathrm{dL}$ & $<1.8$ & $<1.8$ \\
\hline 24-h urinary free cortisol, $\mu \mathrm{g} /$ day & $3.5-45$ & 40.1 \\
\hline 24-h urinary free cortisol, $\mu$ g/day & $3.5-45$ & 40.8 \\
\hline 24-ho urinary free cortisol, $\mu \mathrm{g} /$ day & $3.5-45$ & 39.5 \\
\hline $\begin{array}{l}\text { 24-h urine metanephrines**, } \\
\mu \mathrm{g} / \text { day }\end{array}$ & $<400$ & 160 \\
\hline $\begin{array}{l}\text { 24-h urine normetanephrine**, } \\
\text { g/day }\end{array}$ & $<900$ & 297 \\
\hline $\begin{array}{l}\text { 24-h urine total metanephrine**, } \\
\mu \mathrm{g} / \text { day }\end{array}$ & $<1300$ & 457 \\
\hline
\end{tabular}

*After 1 mg overnight dexamethasone suppression test; ** when hypertensive. 

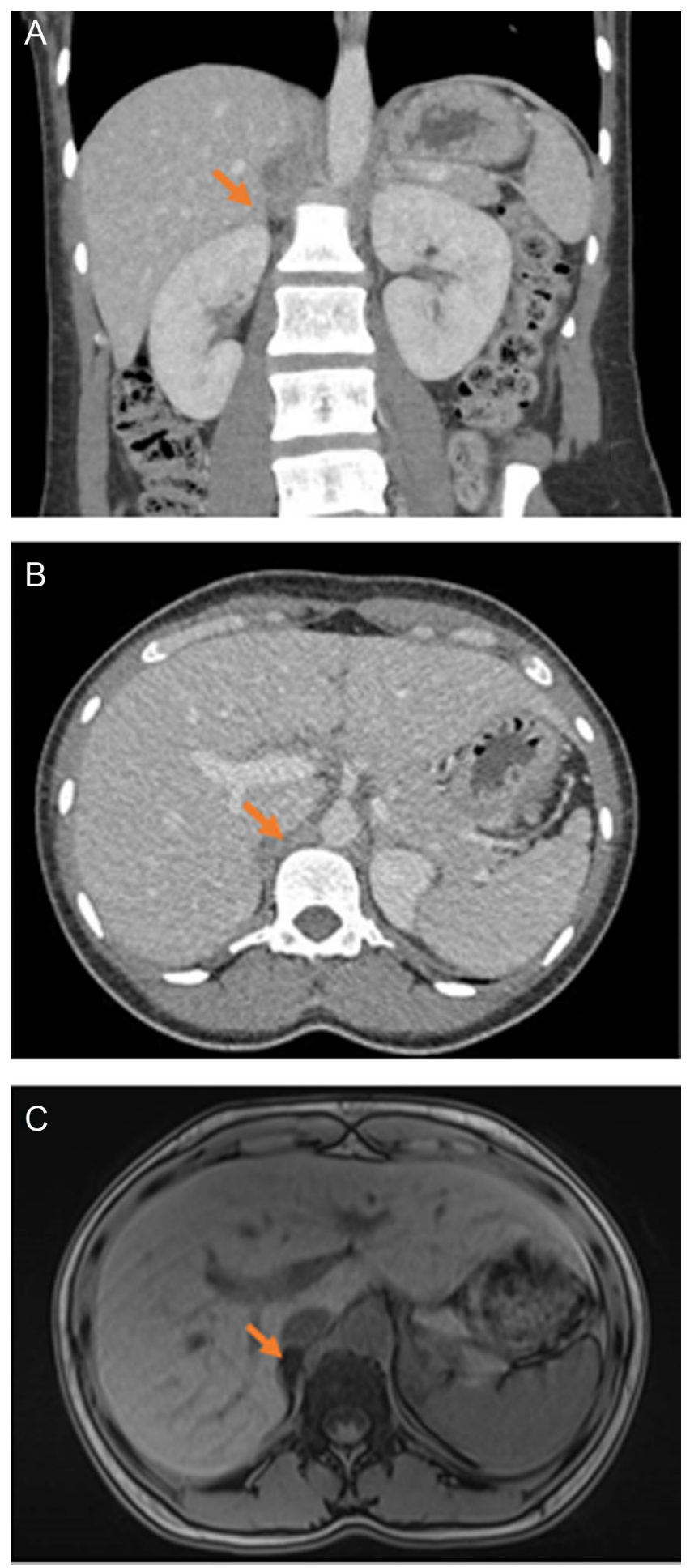

Figure 1

Imaging of the adrenal glands. Non-contrast CT adrenal of the patient: (A) Coronal view. (B) Axial view. (C) MRI of the adrenal glands. Arrow points to the adrenal adenoma. noted to be spontaneously and unintentionally pregnant with an estimated gestational age (EGA) of 5 weeks. Her treatment was switched to hydralazine $50 \mathrm{mg}$ every $8 \mathrm{~h}$ and labetalol $200 \mathrm{mg}$ twice daily with initial control of her hypertension. However, 2 weeks later, the patient's blood pressure was noted to be persistently elevated with blood pressure readings necessitating the addition of nifedipine to her pharmacotherapy and eventual increase in her labetalol dose to $300 \mathrm{mg}$ in the morning and 400 $\mathrm{mg}$ in the evening. These medical treatment measures failed to normalize her blood pressure with systolic blood pressure $>150 \mathrm{mmHg}$ and diastolic blood pressure $>90$ mmHg. The patient was counselled about her treatment options and she elected to pursue laparoscopic unilateral right adrenalectomy. The operation was challenging given that the right adrenal gland was densely adherent to the liver and the inferior vena cava and was positioned high in the retroperitoneum. Ultimately, the patient had right adrenalectomy with clear margins.

\section{Outcome and follow-up}

In the immediate post-operative period, the patient was weaned from labetalol $400 \mathrm{mg}$ twice daily to $150 \mathrm{mg}$ twice daily and her blood pressure and serum potassium gradually improved. At the next post-operative follow-up visit (EGA 23 weeks), when she was off all anti-hypertensive medications, her blood pressure was within the normal range. At EGA of 35 weeks, the patient underwent delivery by cesarean section due to intrauterine growth retardation. She was pre-emptively started on labetalol but remained normotensive and was weaned off this medication by 6 weeks postpartum.

\section{Discussion}

Chronic hypertension affects $1.8 \%$ of all pregnancies and fewer than 100 cases of secondary hypertension from PA have been reported to date (1). The diagnosis of PA in pregnancy poses a unique challenge since the placenta upregulates the secretion of renin and angiotensinogen, which in turn leads to a higher secretion of aldosterone and sodium retention (Fig. 2). Further, a systematic review reported that PA occurrence in pregnancy may only be identified postpartum as persistent hypertension (3).

PA may behave variably in pregnancy, that is, the degree of PA may improve or worsen in pregnancy. This is because, in some women, the anti-mineralocorticoid activity of progesterone may attenuate the effects of aldosterone; whereas in other women the increased 


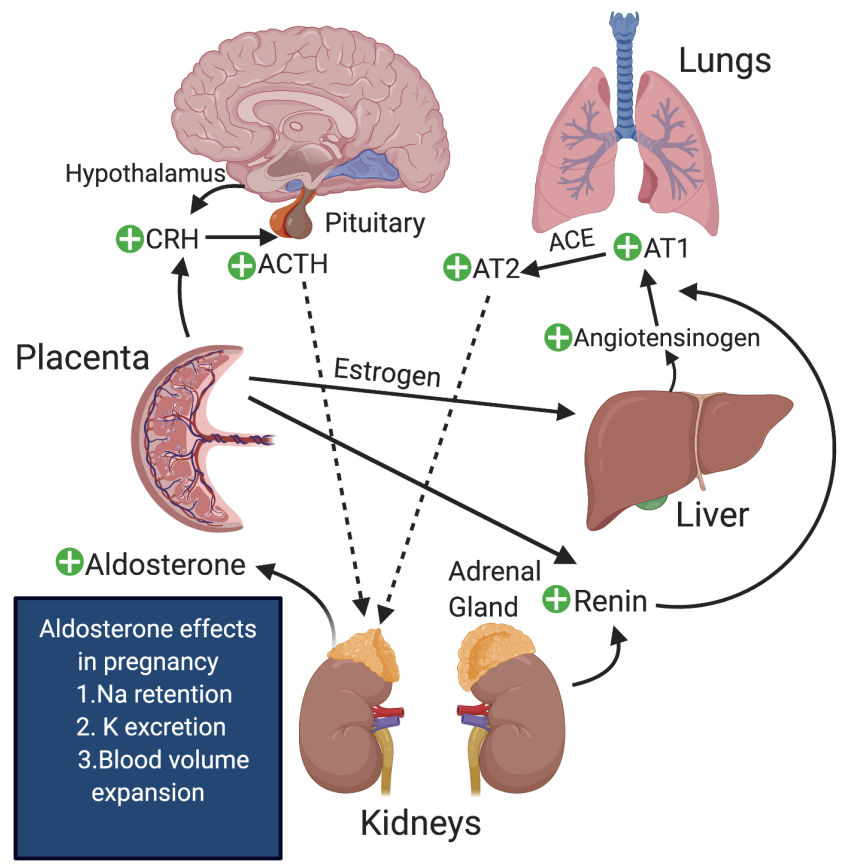

Figure 2

Physiological changes in the renin-angiotensin-aldosterone system in pregnancy. Courtesy of Skand Shekhar, NICHD, NIH.

activation of the renin-angiotensin-aldosterone system may cause increased secretion of aldosterone, which may exacerbate the hypokalemia and hypertension. Furthermore, increased expression of $\mathrm{LH}$ receptor and the gonadotropin-releasing hormone receptor in aldosteronomas may trigger aldosterone release with worsening clinical features in pregnancy $(4,5)$.

Of the previously reported cases of PA in pregnancy, the majority delivered before term $(1,6,7,8,9,10)$. Other notable adverse associations of PA in pregnancy include intrauterine growth retardation and intrauterine fetal demise (1). Our patient underwent a preterm cesarean section in the setting of intrauterine growth retardation reinforcing the association between PA and pregnancy complications. There is recent evidence of the presence of unique adrenal CTNNB1 pathogenic variants in pregnant women who developed PA (5). Our patient developed PA pre-pregnancy. Genetic and immunohistochemical analyses of her aldosteronoma were not pursued.

While non-pregnant reference ranges for plasma aldosterone concentration and plasma renin activity cannot be used in pregnancy due to the aforementioned physiology, the first step in the detection of PA in pregnancy remains the measurement of plasma aldosterone concentration and plasma renin activity or direct renin concentration (11). Alternatively, measurement of 24-h urine sodium and aldosterone (on a liberal sodium diet) can be performed. Malha and colleagues have proposed a cutoff of plasma renin activity of less than $4 \mathrm{ng} / \mathrm{mL} / \mathrm{h}$ instead of less than $1 \mathrm{ng} / \mathrm{mL} / \mathrm{h}$ as in non-pregnant patients (12). Notably, in normokalemic patients with a positive initial screening test, confirmatory testing is advisable. It is important to note that our patient's triad of hypokalemia, elevated plasma aldosterone concentration of greater than $20 \mathrm{ng} / \mathrm{dL}$ and suppressed plasma renin activity (or direct renin concentration equivalent) of less than $1 \mathrm{ng} /$ $\mathrm{mL} / \mathrm{h}$ confirms PA without the need for additional testing. However, our patient was enrolled in a research protocol and, for that reason, additional testing (including saline suppression test and lateralization studies) was performed per protocol. The patient had another notable finding of hypercarbia, which can be seen in PA due to hydrogen excretion from the kidneys. In general, confirmatory testing with saline suppression test or subtype classification with adrenal venous sampling are also not recommended during pregnancy given the risks of iatrogenic volume overload and radiation exposure, respectively. Fortunately, most pregnancies occur in females less than 35-40 years of age, an age cutoff used by the Endocrine Society to forego adrenal venous sampling (11).

Medical management of PA in non-pregnant female patients is achieved by primarily using mineralocorticoid receptor antagonists (MRA) such as spironolactone or eplerenone (13). Given the teratogenic potential of these agents, options for medical management during pregnancy include beta blockers, calcium channel blockers, hydralazine and alpha blockers, which were employed by us in an attempt to control this patient's blood pressure. Some suggest eplerenone as the MRA of choice if an MRA needs to be used in pregnancy (13, 14). However, if hypertension and hypokalaemia remain suboptimally controlled, then surgical intervention such as unilateral adrenalectomy is indicated.

Our patient had pre-pregnancy confirmation of unilateral PA, which aided in choosing a surgical option. This is generally not available to pregnant patients with PA since confirmatory/localization testing is contraindicated. In some reports, six cases of successful adrenalectomy in PA have been reported, of whom five had preterm delivery, similar to our case $(1,2,15)$. One patient who refused surgery in the setting of uncontrolled hypertension and an adrenal lesion had an unfavourable fetal outcome (16). MRI of the abdomen is the radiographic modality of choice to identify the adrenal anatomy and the presence of adrenal tumors before proceeding with surgery. Although most adrenalectomies can be deferred until after delivery, surgeries that must be performed during pregnancy are 
reserved for the second trimester for the best outcomes (17). Indications for adrenalectomy during pregnancy include patient preference, adrenocortical cancer, or severe or poorly controlled hypertension (as in our case).

In conclusion, while PA is rare in pregnancy, it is the most common cause of secondary hypertension with unique diagnostic and therapeutic challenges. If not optimally treated, PA is associated with poor maternal and fetal outcomes.

\section{Declaration of interest}

Constantine Stratakis holds patents on the PRKAR1A, PDE11A, and the GPR101 genes and his laboratory is the recipient of past and ongoing research grant support from Pfizer, Inc.

\section{Funding}

This work was supported by the research project Z01-HD008920 (Principal Investigator: Constantine A Stratakis) of the Intramural Research Program of the Eunice Kennedy Shriver National Institute of Child Health \& Human Development (NICHD), National Institutes of Health (NIH), Bethesda, MD, USA.

\section{Patient consent}

Written informed consent has been obtained from the patient for publication of the submitted article and accompanying images.

\section{Author contribution statement}

S S drafted the manuscript, obtained images, drafted tables and approved the final version. $\mathrm{R} \mathrm{H}$ drafted the manuscript, cared directly for the patient and approved the final version. C K drafted the manuscript, conceptualized the draft and approved the final version. C A S and F H S conceptualized the draft, oversaw the writing, cared for the patient and approved the final version.

\section{References}

1 Affinati AH \& Auchus RJ. Endocrine causes of hypertension in pregnancy. Gland Surgery 20209 69-79. (https://doi.org/10.21037/ gs.2019.12.04)

2 Landau E \& Amar L. Primary aldosteronism and pregnancy. Annales d'Endocrinologie 201677 148-160. (https://doi.org/10.1016/j. ando.2016.04.009)

3 Kilmartin C, Opu T, Podymow T \& Dayan N. Primary hyperaldosteronism presenting as persistent postpartum hypertension: illustrative case and systematic review. Obstetric Medicine 201912 190-195. (https://doi.org/10.1177/17534 95X18772999)
4 Gagnon N, Cáceres-Gorriti KY, Corbeil G, El Ghoyareb N, Ludwig N, Latour M, Lacroix A \& Bourdeau I. Genetic characterization of GnRH/LH-responsive primary aldosteronism. Journal of Clinical Endocrinology and Metabolism 2018103 2926-2935. (https://doi. org/10.1210/jc.2018-00087)

5 Teo AE, Garg S, Shaikh LH, Zhou J, Karet Frankl FE, Gurnell M, Happerfield L, Marker A, Bienz M, Azizan EA, et al. Pregnancy, primary aldosteronism, and adrenal CTNNB1 mutations. New England Journal of Medicine 2015373 1429-1436. (https://doi. org/10.1056/NEJMoa1504869)

6 Morton A. Primary aldosteronism and pregnancy. Pregnancy Hypertension 20155 259-262. (https://doi.org/10.1016/j. preghy.2015.08.003)

7 Okawa T, Asano K, Hashimoto T, Fujimori K, Yanagida K \& Sato A. Diagnosis and management of primary aldosteronism in pregnancy: case report and review of the literature. American Journal of Perinatology 200219 31-36. (https://doi.org/10.1055/s-2002-20170)

8 Eguchi K, Hoshide S, Nagashima S, Maekawa T, Sasano H \& Kario K. An adverse pregnancy-associated outcome due to overlooked primary aldosteronism. Internal Medicine 201453 2499-2504. (https://doi. org/10.2169/internalmedicine.53.2762)

9 Albiger NM, Sartorato P, Mariniello B, Iacobone M, Finco I, Fassina A \& Mantero F. A case of primary aldosteronism in pregnancy: do $\mathrm{LH}$ and GNRH receptors have a potential role in regulating aldosterone secretion? European Journal of Endocrinology 2011164 405-412. (https://doi.org/10.1530/EJE-10-0879)

10 Shigematsu K, Yamaguchi N, Nakagaki T \& Sakai H. A case of unilateral adrenal hyperplasia being difficult to distinguish from aldosterone-producing adenoma. Experimental and Clinical Endocrinology and Diabetes 2009117 124-128. (https://doi. org/10.1055/s-2008-1078737)

11 Funder JW, Carey RM, Mantero F, Murad MH, Reincke M, Shibata H, Stowasser M \& Young WF. The management of primary aldosteronism: case detection, diagnosis, and treatment: an Endocrine Society clinical practice guideline. Journal of Clinical Endocrinology and Metabolism 2016101 1889-1916. (https://doi. org/10.1210/jc.2015-4061)

12 Malha L \& August P. Secondary hypertension in pregnancy. Current Hypertension Reports 201517 53. (https://doi.org/10.1007/s11906015-0563-z)

13 Riester A \& Reincke M. Progress in primary aldosteronism: mineralocorticoid receptor antagonists and management of primary aldosteronism in pregnancy. European Journal of Endocrinology 2015 172 R23-R30. (https://doi.org/10.1530/EJE-14-0444)

14 Gunganah K, Carpenter R \& Drake WM. Eplerenone use in primary aldosteronism during pregnancy. Clinical Case Reports 20164 81-82. (https://doi.org/10.1002/ccr3.355)

15 Nursal TZ, Caliskan K, Ertorer E, Parlakgumus A \& Moray G. Laparoscopic treatment of primary hyperaldosteronism in a pregnant patient. Canadian Journal of Surgery 200952 E188-E190.

16 Kreze Jr A, Kothaj P, Dobakova M \& Rohon S. Primary aldosteronism caused by aldosterone-producing adenoma in pregnancy complicated by EPH gestosis. Wiener Klinische Wochenschrift 1999111 855-857.

17 Kamoun M, Mnif MF, Charfi N, Kacem FH, Naceur BB, Mnif F, Dammak M, Rekik N \& Abid M. Adrenal diseases during pregnancy: pathophysiology, diagnosis and management strategies. American Journal of the Medical Sciences 2014347 64-73. (https://doi. org/10.1097/MAJ.0b013e31828aaeee)

Received in final form 21 June 2020

Accepted 13 July 2020 